\title{
DETERMINANTS OF LAPLACIANS ON THE SPACE OF CONICAL METRICS ON THE SPHERE
}

\author{
HALA KHURI KING
}

\begin{abstract}
On a compact surface with smooth boundary, the determinant of the Laplacian associated to a smooth metric on the surface (with Dirichlet boundary conditions if the boundary is nonempty) is a well-defined isospectral invariant. As a function on the moduli space of such surfaces, it is a smooth function whose boundary behavior in certain cases is well understood; see [OPS and $\mathrm{K}$ ]. In this paper, we restrict ourselves to a certain class of singular metrics on closed surfaces called conical metrics. We show that the determinant of the associated Laplacian is still well defined and that it is a real analytic function on a suitably restricted subset of the space of conical metrics on the sphere.
\end{abstract}

\section{INTRODUCTION}

Let $\Sigma$ denote a compact surface without boundary. Let $\sigma$ be a smooth metric on $\Sigma$ and $\Delta$ be its Laplace-Beltrami operator acting on functions. Let $0=\lambda_{0}<\lambda_{1} \leq \lambda_{2} \cdots$ denote the eigenvalues of $\Delta$. Then for an orthonormal basis of functions $\left\{u_{j}\right\}$ we have $\Delta u_{j}+\lambda_{j} u_{j}=0$. We recall that the determinant of the Laplacian, $\operatorname{det}^{\prime} \Delta$, is formally defined as

$$
\operatorname{det}^{\prime} \Delta=\prod_{j=1}^{\infty} \lambda_{j},
$$

and that to give meaning to this product we use the standard zeta regularization. We introduce the zeta function

$$
Z(s)=\sum_{j=1}^{\infty} \lambda_{j}^{-s}
$$

in terms of which

$$
\operatorname{det}^{\prime} \Delta=\exp \left(-Z^{\prime}(0)\right)
$$

It follows from the asymptotic distribution of the $\lambda_{j}$ 's, given by Weyl's law, that $Z(s)$ defines an analytic function for $\mathfrak{R}(s)>1$. To study the analytic continuation of $Z(s)$, we note that since the gamma function satisfies

$$
\Gamma(s) a^{-s}=\int_{0}^{\infty} e^{-a t} t^{s-1} d t
$$

Received by the editors August 17, 1990 and, in revised form, March 15, 1991.

1991 Mathematics Subject Classification. Primary 58G25.

Key words and phrases. Conical metrics, determinants of Laplacians, spectral geometry of conical metrics. 
we have for $\mathfrak{R}(s)>1+\varepsilon$,

$$
\Gamma(s) \sum_{j=1}^{\infty} \lambda_{j}^{-s}=\sum_{j=1}^{\infty} \Gamma(s) \lambda_{j}^{-s} .
$$

Thus, for $\mathfrak{R}(s)$ large we can write

$$
Z(s)=\frac{1}{\Gamma(s)} \int_{0}^{\infty} \operatorname{TR}\left(e^{\Delta t}-\frac{1}{A}\right) t^{s} \frac{d t}{t},
$$

with $A$ being the area of $(\Sigma, \sigma)$.

It is easy to see that

$$
\int_{1}^{\infty}\left(\sum_{j=1}^{\infty} e^{-\lambda_{j} t}\right) t^{s-1} d t
$$

can be analytically continued in $s$, to all of $\mathbf{C}$. Also as is well known, see [MS], the kernel of $e^{\Delta t}$ has the following expansion as $t \rightarrow 0$;

$$
\sum_{j=0}^{\infty} e^{-\lambda_{j} t} u_{j}^{2}(x)=\frac{1}{4 \pi t}+\frac{K(x)}{12 \pi}+O(t),
$$

where $K(x)$ is the Gaussian curvature of $\Sigma$ at $x$. Integrating these local invariants over $\Sigma$ yields as $t \rightarrow 0$,

$$
\operatorname{TR}\left(e^{\Delta t}\right)=\frac{A}{4 \pi t}+\frac{\chi(\Sigma)}{6}+O(t),
$$

where $\chi(\Sigma)$ denotes the Euler characteristic of $\Sigma$. It follows that

$$
Z(s)=\frac{1}{\Gamma(s)}\left\{\frac{A}{4 \pi(s-1)}+\left(\frac{\chi(\Sigma)}{6}-1\right) \frac{1}{s}+\text { analytic in } s\right\}
$$

in the region $\mathfrak{R}(s)>-1$. Hence $Z$ has an analytic continuation as a meromorphic function, is regular at $s=0$ and (1.2) makes sense.

In case $\Sigma$ has smooth boundary, we subject $\Delta$ to Dirichlet boundary conditions and use the analogue of (1.5) to show that (1.2) still makes sense. We note that to define $\operatorname{det}^{\prime} \Delta$ using (1.2) we needed the discreteness of the spectrum, $\operatorname{spec}(\Delta)$, the asymptotic distribution of the eigenvalues and some known results about the small time behavior of the heat kernel.

From here on $\Sigma$ will denote a closed surface of genus $p$. Let $\left\{P_{1}, \ldots, P_{n}\right\}$ be $n$ distinct points on $\Sigma$ with $n \geq 3$ for $p=0$ and $n \geq 1$ for $p \geq 1$. A conical metric on $\Sigma$ with vertices $\left\{P_{1}, \ldots, P_{n}\right\}$ and exponents $\left\{a_{1}, \ldots, a_{n}\right\}$ is a flat metric on $\Sigma \backslash\left\{P_{1}, \ldots, P_{n}\right\}$ such that in the neighborhood of each $P_{i}$ there are isothermal coordinates so that the line element takes the form

$$
d s=|w|^{a_{i}}|d w|
$$

in $0<|w|<\delta_{i}$, for some $\delta_{i}>0$, where
(a) $a_{i}>-1$,
(b) $\sum_{i=1}^{n} a_{i}=2 p-2$. 
We need condition $(1.8(a))$ to ensure that the line element (1.7) is integrable on smooth curves through the vertices and condition (1.8(b)) follows the GaussBonnet theorem. We shall see that we can still use (1.2) to define $\operatorname{det}^{\prime} \Delta$, when $\Delta$ is the Laplacian associated to a conical metric on $\Sigma$.

Next we say two conical metrics $\sigma_{1}$ on $\Sigma \backslash\left\{P_{1}, \ldots, P_{n}\right\}$ and $\sigma_{2}$ on $\Sigma \backslash\left\{Q_{1}, \ldots, Q_{n}\right\}$ are equivalent if there is a sense-preserving diffeomorphism $f$ onto $\Sigma$ onto itself mapping vertices to vertices for which $\sigma_{2}=f^{*} \sigma_{1}$. The space of conical metrics on $\Sigma, \mathscr{C}_{n}$, is defined to be

$$
\mathscr{C}_{n}=\{\text { conical metrics }\} / \operatorname{Diff}^{+}(\Sigma),
$$

with the action of the diffeomorphism group as above. Let $\mathscr{C}_{n}^{*}$ be the subset of $\mathscr{C}_{n}$ consisting of those metrics with fixed exponents. We show that, for $\Sigma=S^{2}$, Theorem. $\operatorname{det}^{\prime} \Delta$ is real analytic on $\mathscr{C}_{n}^{*}$.

Acknowledgment. This paper is part of my 1990 Stanford thesis. I am grateful to Peter Sarnak, my advisor, for his guidance and support.

\section{Conic Laplacian}

Let $\sigma$ be a conical metric on $\Sigma$ with vertices $\left\{P_{1}, \ldots, P_{n}\right\}$ and exponents $\left\{a_{1}, \ldots, a_{n}\right\}$. In geodesic polar coordinates, this metric takes the form

$$
d s^{2}=d r^{2}+\left(1+a_{i}\right)^{2} r^{2} d \theta^{2}, \quad r=|w|^{1+a_{i}} /\left(1+a_{i}\right),
$$

near $P_{i}$. This is a special case of a cone-like singularity of a manifold where the metric takes the more general form

$$
\sigma=d r^{2}+r^{2} \sigma_{N}(r), \quad 0 \leq r<\varepsilon,
$$

with a smooth family of nonsingular metrics $\sigma_{N}(r)$ on a smooth compact manifold $N^{m}$ without boundary, referred to as the cross section of the cone, and $\sigma_{N}(r)$ constant for small $r$. In this special case, $N$ is the circle $S_{1+a_{i}}^{1}$ of radius $1+a_{i}$. The spectral geometry of Riemannian spaces $Y$ with cone-like singularities as such has been studied by Cheeger [C], Nagase [N], and Brüning-Seeley [BS]. Melrose [M] has also studied the spectral geometry of proper conic metrics defined by the existence of a defining function $r$, on a compact manifold with boundary, in terms of which

$$
\sigma=r^{2 s}\left((d r / r)^{2}+h\right),
$$

where $h$ is a $C^{\infty}$-symmetric 2-tensor. We note that by setting $w=r e^{i \theta},(1.7)$ becomes a special case of (2.3) with $s=1+a_{i}$ and $h=d \theta^{2}$.

Let $X=\Sigma \backslash\left\{P_{1}, \ldots, P_{n}\right\}$ and $H(X)$ denote the completion of

$$
\left\{u \in C_{0}^{\infty}(X):\|u\|_{1}^{2}=\int_{X}\left(|\nabla u|^{2}+|u|^{2}\right) d V<\infty\right\},
$$

in the metric induced by $\|u\|_{1}^{2}=\|u\|^{2}+\|\nabla u\|^{2}$. On $H(X)$ we consider the symmetric bilinear form given by

$$
q(u, v)=\int_{X}(\nabla u . \nabla v) d V
$$

for $u, v \in H(X)$. Then $q$ is a semibounded closed quadratic form. This gives $q$ as the quadratic form of a unique selfadjoint operator $-\Delta$, i.e.,

$$
(-\Delta u, v)=q(u, v),
$$

where $u, v \in H(X)$. 
In the general case, with $Y$ described as above, one has

Theorem 2.1 [C, N]. If $m=\operatorname{dim} N$ is odd, then the collection of eigenfunctions $\phi$ such that $\phi, d \phi$, and $\Delta \phi \in L^{2}$ determines a complete orthonormal basis of $L^{2}$. The eigenspaces are finite dimensional and the eigenvalues satisfy $0 \leq \lambda_{1} \leq$ $\lambda_{2} \cdots \rightarrow \infty$. Furthermore,

$$
N(\lambda) \sim \frac{\operatorname{vol}(Y) \lambda^{(m+1) / 2}}{(4 \pi)^{(m+1) / 2} \Gamma((m+1) / 2+1)}, \quad \text { as } \lambda \uparrow \infty,
$$

where $N(\lambda)=\left|\left\{j \geq 1: \lambda_{j} \leq \lambda\right\}\right|$.

The proof of this theorem (and the corresponding statement if $\operatorname{dim} N$ is even) uses as a main technique the method of separation of variables and the solutions of Bessel equations with a regular singularity at $r=0$. In the special case of the conic Laplacian on $S^{2}$, the discreteness of $\operatorname{spec}(\Delta)$ is pretty straightforward:

Lemma 2.2. Let $\Delta$ be the Laplacian associated with a conical metric $\sigma$ on $S^{2}$. Then $\operatorname{spec}(\Delta)$ is discrete.

Proof. Let $\sigma$ be a conical metric on $S^{2}$ with vertices $\left\{P_{1}, \ldots, P_{n}\right\}$ and exponents $\left\{a_{1}, \ldots, a_{n}\right\}$. Let $B_{i}$ be the ball centered at $P_{i}$ with radius $r_{i}$. Without loss of generality we assume $r_{i}=1$. Let $X=S^{2} \backslash\left\{P_{1}, \ldots, P_{n}\right\}$. To show $\operatorname{spec}(\Delta)$ is discrete, we need only show

$$
\mathbf{F}_{X}=\left\{u \in C^{\infty}(X): \int_{X}\left(|\nabla u|^{2}+u^{2}\right) d V \leq 1\right\}
$$

is precompact in $L^{2}(X)$ : see [RS].

We show first that for any real number $\delta$, the set

$$
\mathbf{F}_{B_{i}}=\left\{u \in C^{\infty}\left(B_{i}\right): \int_{B_{i}}\left(|\nabla u|^{2}+u^{2}\right) d V \leq \delta, u=0 \text { on } \partial B_{i}\right\}
$$

is precompact in $L^{2}\left(B_{i}\right)$. For notational convenience, we drop the subscript $i$. On $B$, the Laplacian associated with $\sigma$, acting on functions, is given by

$$
\Delta=r^{-2 a-2}\left[r \partial_{r}+r^{2} \partial_{r}^{2}+\partial_{\theta}^{2}\right] .
$$

Consider on $B$ the eigenvalue problem

$$
\begin{aligned}
\Delta u+\lambda u & =0, \\
u & =0 \quad \text { on } \partial B .
\end{aligned}
$$

Set $u(r, \theta)=f(r) g(\theta)$ and $\alpha=a+1$. Then (2.9) is equivalent to

$$
\begin{aligned}
r^{2}\left(f^{\prime \prime}+f^{\prime} / r+\lambda r^{2 \alpha} f\right) / f & =-g^{\prime \prime}(\theta) / g(\theta), \\
f(1) & =0 .
\end{aligned}
$$

The first equation in (2.10) gives $g^{\prime \prime}(\theta) / g(\theta)=-m^{2}$, where $m$ is a nonnegative integer. Thus $g(\theta)=\alpha \cos m \theta+\beta \sin m \theta$ for some $\alpha, \beta$. Set $y=f(r)$. Then

$$
y^{\prime \prime}+(1 / r) y^{\prime}+\left(\lambda r^{2 \alpha}-m^{2} / r^{2}\right) y=0 .
$$

The solution of equation (2.11) is given by

$$
y=c_{1} J_{\nu}\left(\sqrt{\lambda} r^{\alpha+1} /(\alpha+1)\right)+c_{2} J_{-\nu}\left(\sqrt{\lambda} r^{\alpha+1} /(\alpha+1)\right),
$$


where $c_{1}$ and $c_{2}$ are constants, $\nu=m /(\alpha+1)$, and $J_{\nu}$ is the Bessel function of order $\nu$. Since $u \in \mathbf{F}_{B}$, we have $c_{2}=0$. Thus (2.12) reduces to

$$
y=c_{1} J_{\nu}\left(\sqrt{\lambda} r^{\alpha+1} /(\alpha+1)\right) \text {. }
$$

The condition $f(1)=0$ implies $\sqrt{\lambda} /(\alpha+1)$ is a zero of the function $J_{\nu}(x)$, which has infinitely many real zeroes $k_{m, j}(j=1,2,3, \ldots)$. We can then write the eigenfunctions in the form

$$
J_{\nu}\left(k_{m, j} r^{\alpha+1}\right)(\alpha \cos m \theta+\beta \sin m \theta),
$$

where $\alpha, \beta$ are arbitrary. Furthermore any function in $C^{2}(B)$ which vanishes on $\partial B$, can be expressed in a convergent series of the form

$$
\sum_{m, j} a_{m, j} J_{\nu}\left(k_{m, j} r^{\alpha+1}\right) \cos m\left(\theta-\theta_{m, j}\right)
$$

In particular, if $\left\{f_{k}\right\} \in \mathbf{F}_{B}$, then having normalized the eigenfunctions, we get

$$
\sum_{m, j}\left(\lambda_{m, j}^{2}+1\right)\left|a_{m, j}^{(k)}\right|^{2} \leq \delta .
$$

Thus for a fixed $m$, there is a sequence $\left\{k_{l}\right\}$ such that $\left\{a_{r, j}^{\left(k_{l}\right)}\right\}$ converges for all $r \leq m$. Using the diagonal process, there exists $\left\{s_{l}\right\}$ such that $\left\{a_{m, j}^{\left(s_{l}\right)}\right\} \rightarrow b_{m, j}$ as $s_{l} \rightarrow \infty$. Set

$$
f(r, \theta)=\sum_{m, j} b_{m, j} J_{\nu}\left(k_{m, j} r^{\alpha+1}\right) \cos m\left(\theta-\theta_{m, j}\right) .
$$

Then $\left\{f_{k}^{\left(s_{l}\right)}\right\} \rightarrow f$ in $L^{2}(B)$ and $\mathbf{F}_{B}$ is precompact in $L^{2}(B)$.

Now we can show that $\mathbf{F}_{X}$ is precompact in $L^{2}(X)$. Let $\left\{\phi_{i}\right\}$ be a partition of unity subordinate to $B_{1}, \ldots, B_{n}, X \backslash U_{i=1}^{n} V_{i}$ where $V_{i}$ is a closed subset of $B_{i}$ and such that $\left|\nabla \phi_{i}\right| \leq k$ for all $i$. Set $A_{0}=X \backslash U_{i=1}^{n} V_{i}, A_{i}=B_{i}$ for $1 \leq i \leq n$. Then for $u \in \mathbf{F}_{X}, u=\sum_{i=0}^{n} \phi_{i} u_{i}$ and for every $0 \leq i \leq n$ we have

$$
\begin{aligned}
\int_{A_{i}}\left(\left|\nabla\left(\phi_{i} u\right)\right|^{2}+\left(\phi_{i} u\right)^{2}\right) d V=\int_{A_{i}}\left(\left|\nabla u \cdot \phi_{i}+u \cdot \nabla \phi_{i}\right|^{2}+\phi_{i}^{2} u_{i}^{2}\right) d V \\
\quad \leq \int_{A_{i}}\left(\phi_{i}^{2}|\nabla u|^{2}+2 \phi_{i} \cdot u \cdot|\nabla u|\left|\nabla \phi_{i}\right|+u^{2}\left|\nabla \phi_{i}\right|^{2}+\phi_{i}^{2} u^{2}\right) d V \\
\quad=\int_{A_{i}} \phi_{i}^{2}\left(|\nabla u|^{2}+u^{2}\right) d V+\int_{A_{i}}\left(\left|\nabla \phi_{i}\right|^{2} u^{2}+2 \phi_{i} u|\nabla u|\left|\nabla \phi_{i}\right|\right) d V \\
\quad \leq \int_{A_{i}}\left(|\nabla u|^{2}+u^{2}\right) d V+\int_{A_{i}}\left(k^{2} u^{2}+k\left(u^{2}+|\nabla u|^{2}\right)\right) d V \\
\leq 1+k^{2}+k
\end{aligned}
$$

whenever $\int_{X}\left(|\nabla u|^{2}+u^{2}\right) d V \leq 1$. Thus given $\left\{g_{n}\right\} \in \mathbf{F}_{X}$, one has $\left\{\phi_{i} g_{n}\right\} \in \mathbf{F}_{A_{i}}$ for all $0 \leq i \leq n$. Using the first result and a diagonal process we can extract a convergent subsequence $\left\{\phi_{i} g_{n_{k}}\right\}$ in $L^{2}(X)$, hence the desired claim.

\section{ASYMPTOTICS OF THE HEAT KERNEL}

The authors in [BS] give a scheme for computing the asymptotics of $\operatorname{TR}\left(e^{-L t}\right)$ as $t \rightarrow 0^{+}$for certain singular operators $L$, a principal example of which is 
the Laplace operator for a manifold with a singularity, where the metric takes the form (2.2). The idea there is to study an appropriate power of the resolvent of $L$ then pass to the heat kernel by a contour integral. The Hilbert space $L^{2}$ on which $\Delta$ acts is a direct sum of an interior part $H_{i}$, consisting of those functions vanishing identically within some distance of the singularity, and a boundary part $H_{b}$, consisting of the complementary space. Since the interior part is well understood, the heart of the problem is thus the construction of the boundary parametrix. Let $W=\{w \in \mathbf{C}:|\arg w|<\pi-\varepsilon\}, \varepsilon>0$. The main result, in our case, is that for any smooth function $\phi$ supported sufficiently near the singular points, we have

$$
\operatorname{TR}\left[\phi\left(\Delta+z^{2}\right)^{-2}\right]=\int_{0}^{\infty} \sigma(x, x z) d x,
$$

where $\sigma(x, w)$ defined on $\mathbf{R} \times W$ is $C^{\infty}$ in $x$, with derivatives analytic in $w$, and such that $\sigma(x, w) \sim \sum_{j} \sigma_{j}(x) w^{\alpha_{j}}$ gives the following expansion as $w \rightarrow \infty$ in $W$ :

$$
\begin{aligned}
\int_{0}^{\infty} \sigma(x, x z) d x \sim \sum_{k \geq 0} z^{-k-1} \int_{0}^{\infty} \frac{w^{k}}{k !} \sigma^{(k)}(0, w) d w \\
+\sum \int_{0}^{\infty}(z x)^{\alpha_{j}} \sigma_{j}(x) d x \\
+\sum_{\alpha_{j}=-1}^{-\infty} z^{\alpha_{j}} \ln z \frac{\sigma_{j}^{\left(-\alpha_{j}-1\right)}(0)}{\left(-\alpha_{j}-1\right) !}
\end{aligned}
$$

Here $\left\{\alpha_{j}\right\}$ is a sequence of complex numbers with $\mathfrak{R}\left(\alpha_{j}\right) \rightarrow-\infty$, and the functions $\sigma^{(k)}(x, w)=\partial_{x}^{k} \sigma(x, w)$ and $\sigma_{j} \in S(\mathbf{R})$ (Schwartz class) are determined by any interior parametrix valid away from the singular point and the divergent integrals are defined by analytic continuation; see [BS].

To give an idea how these asymptotic expansions arise, the Laplacian in the neighborhood of a vertex $P_{i}$ is given by

$$
\Delta=-\partial_{r}^{2}-r^{-1} \partial_{r}+r^{-2} \Delta_{N}
$$

where $\Delta_{N}$ is the Laplacian on $N=S_{1+a_{i}}^{1}$. The change of variables $f \rightarrow \sqrt{r} f$ transforms $\Delta$ into

$$
\Delta=-\partial_{r}^{2}+r^{-2}\left(\Delta_{N}-1 / 4\right) .
$$

Thus on $H_{b}, \Delta$ is given by

$$
-\partial_{r}^{2}+r^{-2} A, \quad A=\Delta_{N}-\frac{1}{4},
$$

where the operator $A$ is unbounded on $L^{2}(N)$, with $A \geq-\frac{1}{4}$. The singular operator, $D_{a}$, which is the Friedrich's extension of $D=-\partial_{r}^{2}+r^{-2} a, a \geq-\frac{1}{4}$, has resolvent with kernel

$$
k_{\nu}(x, y, z)=(x y)^{1 / 2} I_{\nu}(x z) K_{\nu}(y z), \quad x \leq y,
$$

where $\nu=\sqrt{a+\frac{1}{4}}$, and $I_{\nu}, K_{\nu}$ are Bessel functions: see [Ca]. If $\phi$ has compact support, then $\phi\left(D_{a}+z^{2}\right)^{-1}$ has finite trace given by an integral as in (3.2) where

$$
\sigma(x, w)=x \phi(x) I_{\nu}(w) K_{\nu}(w)
$$


has an expansion in terms of $w^{-1}, w^{-2}, \ldots$ as $w \rightarrow \infty$ in any open sector $|\arg w|<\pi / 2-\varepsilon$. A similar expansion for the trace of $\phi\left(\Delta+z^{2}\right)^{-2}$, can be obtained by using the kernel of $\left(D_{a}+z^{2}\right)^{-2}, a \in \operatorname{spec}(\Delta)$, which is given on the diagonal by

$$
k_{\nu}^{2}(x, x, z)=\left(-\frac{1}{2 z} \frac{\partial}{\partial z}\right)\left(x I_{\nu}(x z) K_{\nu}(x z)\right) .
$$

Let $\gamma$ be a cut-off function supported sufficiently close to the singular point $x=0$, such that $\Delta=\Delta_{b}$ on $\operatorname{supp} \gamma$ and $\gamma(x) \equiv 1$ for small $x$. Then, as shown in [BS], TR $\left(\gamma e^{-\Delta t}\right)$ has an asymptotic expansion, as $t \rightarrow 0$, in $t^{(k-3) / 2}$, $t^{(-4-a) / 2}$, and $t^{(-4-a) / 2} \log t$. Here $a \leq-1$ and $k \geq 3$ are integers. Going back to (1.2) and (1.6), we observe that as far as $\operatorname{det}^{\prime} \Delta$ is concerned the terms of interest are the constant term, $c_{0}$, and the coefficient of $\log t, c_{1}$, in this asymptotic expansion. Problems arise when $c_{1} \neq 0$ since then $Z(s)$ has a first order pole at $s=0$ :

$$
\int_{0}^{1} t^{s-1} \log t d t=\lim _{\varepsilon \rightarrow 0} \int_{\varepsilon}^{1} t^{s-1} \log t d t=\lim _{\varepsilon \rightarrow 0}\left[\frac{-\varepsilon^{s} \log \varepsilon}{s}-\frac{1}{s^{2}}\right]=-\frac{1}{s^{2}} .
$$

Set

$$
\zeta(s)=\sum_{a \in \operatorname{spec}(A)} \nu(a)^{-s},
$$

where $\nu(a)=\sqrt{a+\frac{1}{4}}$. Let $B_{k}$ denote the $k$ th Bernoulli number, and $\operatorname{Res}_{k} f\left(z_{0}\right)$ denote the coefficient of $\left(z-z_{0}\right)^{-k}$ in the Laurent expansion of a meromorphic function $f$ at $z_{0}$. Then $c_{0}$ and $c_{1}$ are given by

Theorem 3.1 [BS].

(a)

$$
\begin{aligned}
c_{0}= & -\frac{1}{2} \operatorname{Res}_{0} \zeta(-1)-\frac{1}{4} \sum_{k \geq 1}(-1)^{k} \frac{B_{k}}{k} \operatorname{Res}_{1} \zeta(2 k-1) \\
& +\frac{\Gamma^{\prime}\left(-\frac{1}{2}\right)}{8 \sqrt{\pi}} \operatorname{Res}_{1} \zeta(-1)+\int_{0}^{\infty} g_{0}(x) d x,
\end{aligned}
$$

(b) $c_{1}=\frac{1}{4} \operatorname{Res}_{1} \zeta(-1)$, where $g_{0}(x)$ satisfies $\lim _{x \rightarrow 0} x g_{0}(x)=-\frac{1}{2} \operatorname{Res}_{1} \zeta(-1)$.

For $N=S_{1+a}^{1}$ and $\sigma_{N}(r)=(1+a)^{2} d \theta^{2}$, if $\beta=1+a$ then the eigenvalues of $A$ are given by $\left\{n^{2} / \beta^{2}-1 / 4\right\}, n \in \mathbf{Z}$, and the zeta function (3.9) is given by

$$
\zeta(s)=2 \sum_{n=1}^{\infty}\left(\frac{n}{\beta}\right)^{-s}=2 \beta^{s} \zeta_{R}(s) .
$$

Since

$$
\zeta_{R}(-1)=-1 / 12, \quad \operatorname{Res}_{1} \zeta_{R}(1)=1, \quad \text { and } \quad B_{1}=-1 / 6,
$$

Theorem 3.1 yields $c_{1}=0$, and

$$
c_{0}=\frac{1}{12 \beta}-\frac{\beta}{12}+\int_{0}^{\infty} g_{0}(x) d x .
$$

Thus we have the regularized interior term $\int_{0}^{\infty} g_{0}(x) d x$, plus singular terms in the zeta function (3.9). One can easily show that if $\sigma$ is flat on $\Sigma_{0} \backslash\left\{P_{1}, \ldots, P_{n}\right\}$ 
then $\int_{0}^{\infty} g_{0}(x) d x=0$. Since $c_{1}=0, Z(s)$ is regular at $s=0$ and we can use (1.2) to define $\operatorname{det}^{\prime} \Delta$.

\section{ReAl ANALYTICITY}

Let $\mathscr{C}_{n}$ denote the space of conical metrics on the sphere with $n$ vertices. Let $\mathscr{C}_{n}^{*}$ denote the subspace consisting of those metrics with fixed exponents. Any point in $\mathscr{C}_{n}$ determines a smooth conformal structure on $S^{2}$. Since there is only one conformal structure on $S^{2}$ determined by the standard round metric $\sigma_{0}$, it follows that each point in $\mathscr{C}_{n}$ has a representative of the form

$$
\sigma=e^{2 \phi} \sigma_{0}
$$

where $\phi$ is smooth and harmonic with respect to $\sigma_{0}$ on $S^{2} \backslash\left\{P_{1}, \ldots, P_{n}\right\}$, for some $P_{1}, \ldots, P_{n}$. We identify $S^{2}$ with $\overline{\mathbf{C}}=\mathrm{C} \cup(\infty)$ with local coordinates $z$ on $C$ and $1 / z$ near $\infty$ and with the standard conformal structure. A conical metric $\sigma$ on $\overline{\mathbf{C}} \backslash\left\{\tau_{1}, \ldots, \tau_{n}\right\}$, with $\tau_{i} \in \overline{\mathbf{C}}$, corresponding to (4.1) may be written as $\sigma=e^{2 \phi}|d z|^{2}$ on $C \backslash\left\{\tau_{1}, \ldots, \tau_{n}\right\}$, and as $\sigma=e^{2 \phi(1 / z)}|d z|^{2} /|z|^{4}$ for $z$ near 0 , using the coordinate $1 / z$ near $\infty$. We shall show

Theorem 4.1. $\operatorname{det}^{\prime} \Delta: \mathscr{C}_{n}^{*} \rightarrow \mathbf{R}$ is real analytic.

Proof. Let us first prove the theorem for the case of four vertices. Let $\sigma$ be a conical metric on $\overline{\mathbf{C}}$ with vertices $\left\{\tau, \tau_{0}, \tau_{1}, \tau_{2}\right\}$ and fixed exponents $\left\{a, a_{0}, a_{1}, a_{2}\right\}$. Assume that $\tau_{i}$ is fixed for $i=0,1,2$ with $\left|\tau_{i}\right|>2$ and $|\tau|<\varepsilon$ for a given $\varepsilon>0$. Rather than work with $\Delta_{\sigma}$, a fixed operator on a varying space, it is more convenient to work with a family of varying operators on a fixed underlying space. To that end, let $f$ be a $C^{\infty}$-function with $f=1$ inside the circle of radius 1 about 0 and $f=0$ outside the circle of radius 2 about 0 . Let $\left\{g_{t}(z)\right\}$ be the flow generated by the vector field $X=f \tau$ with $0 \leq t \leq 1$. Then $\phi_{\tau}(z)=g_{1}(z)=z+f \tau$ is a diffeomorphism of $\overline{\mathbf{C}}$ fixing $\tau_{i}$ and mapping the disk of radius 1 about 0 conformally to the disk of radius 1 about $\tau$ with $\phi_{\tau}(0)=\tau$. Under pullback of metrics, we get a family of metrics $\left\{\sigma_{\tau}\right\}$ on $\overline{\mathbf{C}}$ with fixed vertices $\left\{0, \tau_{0}, \tau_{1}, \tau_{2}\right\}$ which is analytic in $\tau$ and fixed in the neighborhood of a vertex.

Near a vertex $\tau$ of a conical metric on surface $\Sigma$, we have Figure 1 .

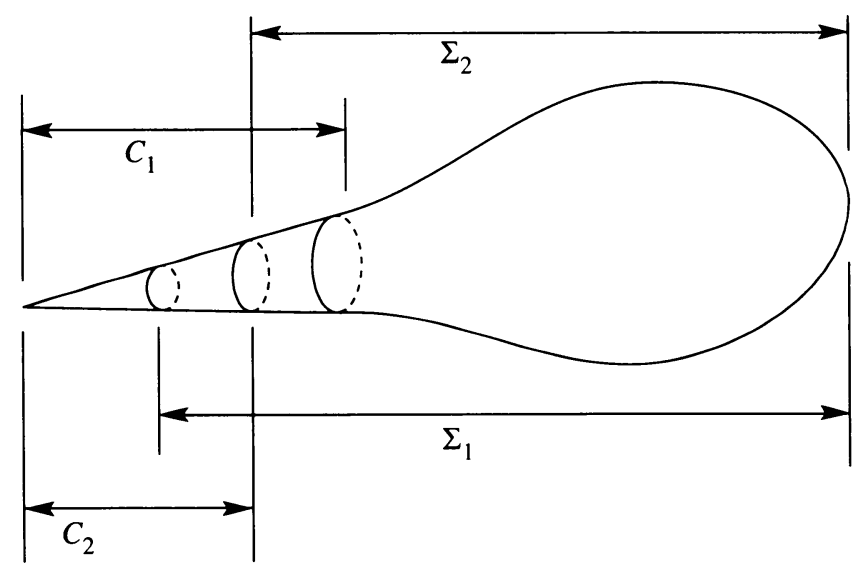

FIGURE 1 
It is shown in [N, Lemma 5.3] that

$$
k_{\tau, \Sigma}(x, x, t)=k_{\tau, \Sigma_{1}}(x, x, t) I_{\Sigma_{2}}+k_{\tau}, C_{1}(x, x, t) I_{C_{2}}+O\left(e^{-\delta / t}\right),
$$

where $k_{\tau, M}$ is the heat kernel on $M, I_{M}$ is the characteristic function on $M$ and $O\left(e^{-\delta / t}\right), \delta>0$ is a term any derivatives of which decrease exponentially when $t \rightarrow 0$. The main idea there is to reconstruct the heat kernel of $\Sigma$, using E. E. Levi's method, from the well-known heat kernel of a compact surface and from the formal representation of the heat kernel involving Bessel functions in a neighborhood of a cone-like singularity. Using estimates of Bessel functions and the uniqueness of the heat kernel, one gets (4.2).

Set $h(\sigma)=-\log \left(\operatorname{det}^{\prime} \Delta\right)$, then

$$
\begin{aligned}
h\left(\sigma_{\tau}\right)= & \left.\frac{d}{d s}\right|_{s=0} \frac{1}{\Gamma(s)}\left[\int_{0}^{1} \int_{\Sigma} k_{\tau, \Sigma}(x, x, t) d x t^{s} \frac{d t}{t}\right] \\
& +\int_{1}^{\infty} \int_{\Sigma} k_{\tau, \Sigma}(x, x, t) d x \frac{d t}{t} .
\end{aligned}
$$

To analyze (4.3) note that we have arranged the problem so that the metric does not change in the neighborhood of a vertex. Thus one parametrix for the Laplacian in a neighborhood of a vertex will work uniformly for all vertices, and the construction of such a parametrix has already been done in [BS]. Away from the vertices, we need a further result. Let $\left\{g_{\tau}\right\}$ be a family of metrics analytic in $\tau$ on a compact surface $M$ with smooth boundary, and $\left\{\Delta_{\tau}\right\}$ be the corresponding family of Laplacians on $L^{2}(M)$. Let $\left\{\phi_{1, \tau}, \phi_{2, \tau}, \ldots\right\}$ be a complete orthonormal basis of $L^{2}(M)$ consisting of eigenfunctions of $\Delta_{\tau}$ with $\phi_{j, \tau}$ having eigenvalue $\lambda_{j, \tau}: 0<\lambda_{1, \tau} \leq \lambda_{2, \tau}, \ldots$. Then $\lambda_{1, \tau}$ is continuous in $\tau$ and is uniformly bounded from below by some constant $c$. Let $k_{\tau}(x, x, t)$ denote the heat kernel on $\left(M, g_{\tau}\right)$. Then

Lemma 4.2. (1) $k_{\tau}(x, x, t)$ is analytic in $\tau$.

(2) Given $T>0,\left|k_{\tau}(x, x, t)\right|=O\left(e^{-c t / 4}\right)$ for all $t \geq T$.

(3) For small $t$ and fixed $\tau_{0}$, the error term in the expansion of $k_{\tau}(x, x, t)$ is uniform in $\tau$ in a neighborhood of $\tau_{0}$.

Proof. We recall the construction of a parametrix for $\Delta$. Let $\varepsilon=\operatorname{inj}(M)$, $B_{\varepsilon}=B(y, \varepsilon)$ for $y \in M$. We introduce geodesic spherical coordinates on $B_{\varepsilon}$ by

$$
x=\exp _{y}(r \xi), \quad 0 \leq r \leq \varepsilon, \xi \in M_{y} \text { with }|\xi|=1 .
$$

Let

$$
S_{k}(x, y, t)=\frac{1}{4 \pi t} \exp \left(\frac{-d^{2}(x, y)}{4 t}\right) \sum_{j=0}^{k} u_{j}(x, y) t^{j},
$$

where $u_{j}(, y): B_{\varepsilon} \rightarrow \mathbf{R}$ satisfy

$$
\begin{aligned}
& u_{0}(y, y)=1 \\
& \partial u_{0} / \partial r+\frac{1}{2}\left(\phi^{\prime} / \phi\right) u_{0}=0, \\
& \partial u_{j} / \partial r+\left[\frac{1}{2} \phi^{\prime} / \phi+j / r\right] u_{j}=\Delta u_{j} / r
\end{aligned}
$$


for $j \geq 1$. Here we use the notation of [Ch, p. 149], where $r \phi\left(\exp _{y} r \xi, y\right)$ is defined as a determinant of a path of linear transformations on the orthogonal space of $\xi$ defined in terms of parallel translation and Jacobi fields along geodesics $\gamma_{\xi}$. The solution to (4.5) is given by

$$
\begin{aligned}
& u_{0}(x, y)=\phi^{-1 / 2}(x, y), \\
& u_{j}(x, y)=u_{0}(x, y) \int_{0}^{1} \tau^{j-1}\left(u_{0} \Delta u_{j-1}\right)\left(\exp _{y} \tau r \xi, y\right) d \tau,
\end{aligned}
$$

for $j \geq 1$. Let $0 \leq \rho \leq 1 \in C^{\infty}(M \times M)$ be equal to 1 on $B_{\varepsilon / 4}$ and 0 on $(M \times M) \backslash B_{\varepsilon / 2}$. A parametrix for $(\Delta-\partial / \partial t)$ on $M$ is then given by $H_{k}=\rho S_{k}$, which satisfies

$$
\left(\Delta_{x}-\partial / \partial t\right) H_{k}=t^{k-1} \exp \left(-d^{2}(x, y) / 4 t\right) G_{k},
$$

with $G_{k} \in C^{\infty}(M \times M \times[0, \infty))$. A fundamental solution to the heat equation is then given by

$$
k(x, x, t)=H_{k}+H_{k} * \sum_{l=1}^{\infty}\left[\left(\Delta_{x}-\frac{\partial}{\partial t}\right) H_{k}\right]^{* l}=H_{k}+H_{k} * F,
$$

where for $0 \leq t \leq T$, one has the estimate

$$
|F(x, y, t)| \leq C t^{k-1} \exp \left(-d^{2}(x, y) / 4 t\right) .
$$

It is well known from the theory of ordinary differential equations [CL] that the solution of an ordinary differential equation, depending continuously (or analytically) on a parameter, depends continuously (or analytically) on the given data. Since geodesics $\gamma:(a, b) \rightarrow M$ are solutions of second degree ordinary differential equations whose coefficients are the Christoffel symbols (which are smooth functions in the coefficients of the metric $g_{\tau}$ ), geodesics on $M$ depend smoothly on $\tau$. Similarly for a parallel field of vectors $v(t)$ along a geodesic or for a Jacobi field $Z$. We can thus conclude that $u_{0, \tau}$ is analytic in $\tau$. Using the iteration formula (4.6) we see that $u_{j, \tau}$ is analytic in $\tau$ for all $j$. This gives (1) in Lemma 4.2.

For the large time behavior, let $T>0$ be given. Then

$$
k_{\tau}(x, x, t)=\sum_{j=1}^{\infty} e^{-\lambda_{j, \tau} t} \phi_{j, \tau}(x) \phi_{j, \tau}(x),
$$

and

$$
\begin{aligned}
\left|\sum_{j=1}^{\infty} e^{-\lambda_{j, \tau} t} \phi_{j, \tau}(x) \phi_{j, \tau}(x)\right| & \leq \sum_{j=1}^{\infty} e^{-\lambda_{j, \tau} t}\left\|\phi_{j, \tau}\right\|_{\infty}^{2} \\
& \leq C \sum_{j=1}^{\infty} e^{-\lambda_{j, \tau} t}\left\|D^{2} \phi_{j, \tau}\right\|_{2}^{2} \leq C \sum_{j=1}^{\infty} e^{-\lambda_{j, \tau} t}\left(\lambda_{j, \tau}\right)^{2},
\end{aligned}
$$

where we used a standard Sobolev inequality [F]. Using the simple fact that $e^{-s} s \leq e^{-s / 2}$ for $s \geq \ln 4$, and the well-known asymptotic distribution of large eigenvalues $\lambda_{j} \sim \alpha j$ as $j \rightarrow \infty$, we can choose $N$ large enough so that for $j \geq N$ we have

$$
\begin{aligned}
e^{-\lambda_{j, \tau} t}\left(\lambda_{j, \tau}\right)^{2} & \leq\left(2 / t^{2}\right) e^{-\lambda_{j, \tau} t / 4}, \\
\lambda_{j, \tau} & \geq \alpha j / 2
\end{aligned}
$$


and

$$
e^{-\left(\alpha j / 2-\lambda_{1, \tau}\right) t / 4} \leq j^{-2}
$$

Then

$$
\sum_{j=1}^{N} e^{-\lambda_{j, \tau} t}\left(\lambda_{j, \tau}\right)^{2} \leq 2 N e^{-\lambda_{1, \tau} t / 4}
$$

and

$$
\begin{aligned}
\sum_{j=N+1}^{\infty} e^{-\lambda_{j, \tau} t}\left(\lambda_{j, \tau}\right)^{2} & \leq 2 \sum_{j=N+1}^{\infty} e^{-\lambda_{j, \tau} t / 4} \leq 2 e^{-\lambda_{1, \tau} t / 4} \sum_{j=N+1}^{\infty} e^{-\mu_{j, \tau} t / 4} \\
& \leq 2 e^{-\lambda_{1, \tau} t / 4} \sum_{j=N+1}^{\infty} e^{-\left(\alpha j / 2-\lambda_{1, \tau}\right) t / 4} \\
& \leq 2 e^{-\lambda_{1, \tau} t / 4} \sum_{j=N+1}^{\infty} j^{-2} \leq B e^{-\lambda_{1, \tau} t / 4}
\end{aligned}
$$

where

$$
\mu_{j, \tau}=\lambda_{j, \tau}-\lambda_{1, \tau}, \quad B=2 \sum_{j=N+1}^{\infty} j^{-2} .
$$

Putting all this together we get

$$
\left|k_{\tau}(x, x, t)\right| \leq C(2 N+B) e^{-\lambda_{1, \tau} t / 4},
$$

hence the desired estimate

$$
\left|k_{\tau}(x, x, t)\right|=O\left(e^{-c t / 4}\right) .
$$

For the small time behavior, let $T>0$ and $\tau_{0}$ be given. Then, for $t \in$ $[0, T]$, the constant $C$ appearing in (4.8) is a function of the volume of $M$, $T$, and $\sup \left|G_{k}\right|$ on $M \times M \times[0, T]$. Using the analyticity of $u_{j, \tau}$ in $\tau$ on the compact surface $M$ we get the uniformity in $\tau$ of $\sup \left|G_{k}\right|$ in a neighborhood of $\tau_{0}$, hence the desired result. Putting together (4.2), (4.3), Lemma 4.2, and the remark preceding it we get Theorem 4.1 for four vertices.

For the general case, we proceed in a similar manner. Let $\sigma$ be a conical metric on $\overline{\mathbf{C}}$ with fixed exponents and with vertices $\left\{0,1, \infty, \tau_{4}, \ldots, \tau_{n}\right\}$. Assume that $\left|\tau_{i}-\tau_{i}^{0}\right|<\varepsilon$ for a given $\varepsilon$ and fixed $\tau_{i}^{0}$. We construct, as before, a diffeomorphism of $\overline{\mathbf{C}}$ fixing $\{0,1, \infty\}$ and mapping a small disk about $\tau_{i}$ conformally onto a small disk about $\tau_{i}^{0}$, with $\tau_{i}$ mapped to $\tau_{i}^{0}$. Under pullback of metrics, we get a family of metrics on $\overline{\mathbf{C}}$ with fixed vertices $\left\{0,1, \infty, \tau_{4}^{0}, \ldots, \tau_{n}^{0}\right\}$ which is analytic in the parameters $\tau_{i}$ and is fixed in the neighborhood of a vertex. The proof is now identical to the one for four vertices. This finishes the proof of the theorem.

\section{BIBLIOGRAPHY}

[BS] J. Brüning and R. Seeley, The resolvent expansion for second order operators, J. Funct. Anal. 73 (1987), 369-429.

[Ca] C. Callias, The heat equation with singular coefficients. I, Comm. Math. Phys. 88 (1983), 357-385. 
[Ch] I. Chavel, Eigenvalues in Riemannian geometry, Academic Press, 1984.

[C] J. Cheeger, On the spectral geometry of spaces with cone-like singularities, Proc. Nat. Acad. Sci. U.S.A. 76 (1979), 2103-2106.

[CL] E. A. Coddington and N. Levinnson, Theory of ordinary differential equations, McGraw-Hill, New York, 1955.

[F] A. Friedman, Partial differential equations, Krieger, 1969.

[K] H. H. Khuri, Heights on the moduli space of Riemann surfaces with circle boundaries, preprint.

[MS] H. P. McKean, Jr. and I. M. Singer, Curvature and eigenvalues of the Laplacian, J. Differential Geom. 1 (1967), 43-69.

[M] R. Melrose, Analysis on manifolds with corners, Lecture notes, M.I.T., 1988.

[N] N. Nagase, The fundamental solution of the heat equation on Riemannian spaces with cone-like singular points, Kodai Math. J. 7 (1984), 382-455.

[OPS] B. Osgood, R. Phillips, and P. Sarnak, Moduli space, heights and isospectral sets of plane domains, Ann. of Math. (2) 129 (1989), 293-362.

[RS] M. Reed and B. Simon, Methods of mathematical physics, vol. 4, Academic Press, 1978.

Department of Mathematics, University of California, Santa Cruz, California 95064

Current address: Natural Science Division, Pepperdine University, 24255 Pacific Coast Highway, Malibu, California 90263 